\section{Relevancia clínica de las interacciones medicamentosas: Propuesta de actualización de la clasificación, acorde con su gravedad y probabilidad}

\section{Clinical relevance of drug interactions: Proposal to update the classification based on the severity and probability of its occurrence}

\section{Sr. Editor:}

Las interacciones medicamentosas son una fuente de variabilidad farmacológica y, por ello, pueden afectar los resultados de la farmacoterapia y generar resultados terapéuticos no deseados, ya sea en no lograr el objetivo del tratamiento (inefectividad) o la aparición de problemas de salud adicionales en el paciente (inseguridad) ${ }^{1}$. En este sentido, el año 2007 publicamos una revisión, en la que se presentó una propuesta de pasos a seguir, para establecer la relevancia clínica de las interacciones medicamentosas, en especial del mecanismo farmacocinético, al igual que una clasificación para dicha relevancia clínica, basada en una combinación de la gravedad del efecto de la interacción en la salud del paciente y la probabilidad de su ocurrencia ${ }^{2}$. Dicha propuesta de clasificación genera cuatro niveles de relevancia clínica: 1 (riesgo muy alto, color rojo), 2 (alto, color naranja), 3 (medio, color amarillo) y 4 (bajo, color verde) (Tabla 1$)^{2}$.

La propuesta de análisis y de clasificación según relevancia clínica ha sido empleada en el análisis y valoración de interacciones de varios grupos farmacológicos, entre ellos antibacterianos ${ }^{3}$, antivirales para hepatitis $\mathrm{C}^{4}$ $\mathrm{y}$ anti-retrovirales $\mathrm{s}^{5-7}$. En el caso de los anti-retrovirales, y específicamente en pacientes con VIH/SIDA, se ha avanzado en la sistematización de la información, incluyendo la información y soporte de la interacción en un programa informático ${ }^{8} \mathrm{y}$, más recientemente, en una aplicación móvil de acceso libre, disponible en Play Store 9 , buscando favorecer la apropiación y utilización de este recurso.
Tabla 1. Niveles de relevancia clínica de las interacciones medicamentosas: combinación de gravedad y probabilidad de ocurrencia ${ }^{2}$

\begin{tabular}{lccc}
\hline Gravedad & Definida & $\begin{array}{c}\text { Probabilidad } \\
\text { Probable }\end{array}$ & Posible \\
\cline { 2 - 4 } Grave & 1 & 1 & 2 \\
Moderada & 2 & 2 & 3 \\
Leve & 3 & 3 & 4 \\
Sin (ausencia de) gravedad* & $5^{*}$ & N/A* $^{*}$ & N/A* \\
\hline
\end{tabular}

*Propuesta de fila y celdas adicionales a incorporar, a la propuesta inicial publicada en 2007. N/A: No aplica.
Adicionalmente, la utilización de dicha clasificación, basada en la combinación de la gravedad del efecto de la interacción en la salud del paciente (grave, moderada y leve) y en la probabilidad de ocurrencia (probada, definida y posible, acorde con el tipo de estudio que documenta dicho efecto $)^{2}$, en diferentes grupos terapéuticos ${ }^{3-7}$, mostró la necesidad de adicionar un nivel nuevo de relevancia clínica (nivel 5) ${ }^{4,7}$. Este nivel 5 se caracteriza por la ausencia de efecto en la salud del paciente (ausencia de gravedad de la interacción), la cual es documentada en meta-análisis, revisiones sistemáticas o ensayos clínicos (probabilidad definida) y, por tanto, con evidencia de la ausencia de interacción clínicamente relevante. Por ello, este nivel 5 corresponde a medicamentos cuya utilización simultánea no genera interacciones relevantes clínicamente, con evidencia documentada. En los casos en los que la ausencia de gravedad de interacción provenga de estudios analíticos o por la descripción de tres o más reportes de casos (probabilidad probable) o por el reporte de menos de tres casos o por recomendaciones de grupos de expertos posible (probabilidad posible), no aplica dicho nivel (Tabla 1).

El conocimiento y utilización de la información de ausencia de interacciones clínicamente relevantes, para una determinada combinación de fármacos, resulta de interés para los clínicos. Por ello, se espera que esta propuesta de actualización de la clasificación inicial ${ }^{2}$ (adición del nivel 5), contribuya a identificar y a utilizar información basada en la evidencia en pro del logro de los mejores resultados en salud posibles con la utilización de medicamentos, tal como se reporta en la revisión de interacciones para anti-retrovirales publicada en el 2019 y en la cual se detallan los medicamentos con evidencia de ausencia de interacciones clínicamente relevantes (Nivel 5)?

\section{Referencias bibliográficas}

1.- Ospina A, Benjumea D M, Amariles P. Problemas de proceso y resultado relacionados con los medicamentos: evolución histórica de sus definiciones. Rev Fac Nac Salud Pública 2011; 29: 329-40.

2.- Amariles P, Giraldo N A, Faus M J. Interacciones medicamentosas: aspectos generales y aproximación para establecer y utilizar su relevancia clínica [Clinical relevance of drug interactions]. Med Clin (Barc) 2007; 129: 27-35. doi: 10.1157/13106681.

3.- Pino-Marín D, Madrigal- Cadavid J, Amariles P. Relevancia clínica de interacciones de antibióticos relacionadas con cambios en la absorción: revisión estructurada. Rev CES Med 2018; 32: 235-49.

4.- Peláez J, Pino Marín D, Álvarez P, González J, Amariles P. Structured review of establishing and evaluating clinical relevance of drug interactions in hepatitis $\mathrm{C}$ virus treatment (Update 2015-2017). Rev Col Gastroenterol 2019; 34: 15976. doi:10.22516/25007440.252. 
5.- Amariles P, Giraldo N A, Faus M J. Interacciones medicamentosas en pacientes infectados con el VIH: aproximación para establecer y evaluar su relevancia clínica. Farm Hosp 2007; 31: 283-302. doi: 10.1016/s11306343(07)75392-8.

6.- Giraldo N A, Amariles P, Pino-Marín D, Faus M J. Relevancia clínica de las interacciones medicamentosas en pacientes infectados con el virus de la inmunodeficiencia humana: actualización 2009-2014. Rev Chilena Infectol 2016; 33 (Suppl 1): 36-53. doi: 10.4067/S071610182016000700005 .

7.- Osorio L, Rivera M, Pino-Marín D E, Giraldo N A, Amariles P. Relevancia clínica de las interacciones medicamentosas en pacientes infectados con el virus de la inmunodeficiencia humana: actualización 2015-2017. Rev Chilena Infectol 2019; 36: 475-89. doi: 10.4067/S0716-10182019000400475.

8.- Giraldo N A, Amariles P, Monsalve M, Faus M J.

Free software to analyse the clinical relevance of drug interactions with antiretroviral agents $\left(\mathrm{SIMARV}^{\circledR}\right)$ in patients with HIV/AIDS. Res Social Adm Pharm 2017; 13: 831-9. doi: 10.1016/j.sapharm.2016.09.002.

9.- Amariles P, Giraldo N, Madrigal-Cadavid J, Granados J. Free InterApp ARV: The subsequent version of SIMARV ${ }^{\mathbb{R}}$ to analyse the clinical relevance of drug interactions with antiretroviral agents in patients with HIV/AIDS. Res Social Adm Pharm 2019; 15: 1376. doi: 10.1016/j. sapharm.2019.07.014.

Pedro Amariles ${ }^{1}$, Juliana Madrigal-Cadavid ${ }^{1}$ y Newar A. Giraldo ${ }^{I}$

${ }^{I}$ Grupo de Investigación Promoción y Prevención Farmacéutica, Universidad de Antioquia, Colombia.

Correspondencia a: pedro.amariles@udea.edu.co 